Supporting Information

\title{
X-ray Photoelectron Spectroscopy and First Principles Calculation of BCN Nanotubes
}

Shin Young Kim, Jeunghee Park, Hyun Chul Choi, Jae Pyung Ahn, Jin Qiang Hou, and Hong Seok Kang*

\section{(1) Survey-scanned XPS of BN, $B_{0.45} \mathrm{C}_{0.1} \mathrm{~N}_{0.45}$, and BN-C NTs}

Figure S1 displays the survey-scanned XPS spectra measured using a variable photon energy in the range of 350-1265 eV, showing the distribution contour of the $\mathrm{B}, \mathrm{C}$, and $\mathrm{N}$ ls peaks of the $\mathrm{BN}, \mathrm{B}_{0.45} \mathrm{C}_{0.1} \mathrm{~N}_{0.45}$, and $\mathrm{BN}-\mathrm{C}$ NTs. The $\mathrm{O}$ peak likely originated mainly from the substrates, with some contribution from the dangling bonds of the graphite layers, such as the $\mathrm{C}=\mathrm{O}$ bonds. In the case of the BN NTs (mixed with CNTs), the $\mathrm{B} / \mathrm{N}$ ratio remains constant at $\sim 1$, as the photon energy increases from 475 to 1265 $\mathrm{eV}$ [Figure S1a]. The C $1 s$ peak originates from the CNTs. The $\mathrm{B}_{0.45} \mathrm{C}_{0.1} \mathrm{~N}_{0.45}$ NTs exhibit the same $\mathrm{C}$ concentration, independently of the photon energy [Figure S1b]. The C concentration was estimated from the area of their $1 s$ peaks, taking into consideration their relative peak sensitivities, and is $0.15 \pm 0.05$ for all photon energies. This $\mathrm{C}$ content is consistent with the value of 0.1 obtained from EELS within the margin of uncertainty of the XPS measurement. As shown in Figure S1c, the C concentration of the BN-C NTs decreases from 0.2 to 0.1 as the photon energy increases from 475 to $912 \mathrm{eV}$. This decrease of the $\mathrm{C}$ content (by a factor of 2), as the average probing depth of the $\mathrm{B}, \mathrm{C}$, 
and $\mathrm{N} 1 s$ electrons increases from 1 to $5 \mathrm{~nm}$, indicates the lower $\mathrm{C}$ content further inside the nanotubes. This result is quite consistent with the EELS data which indicate that the C outerlayers sheath the BN NTs.

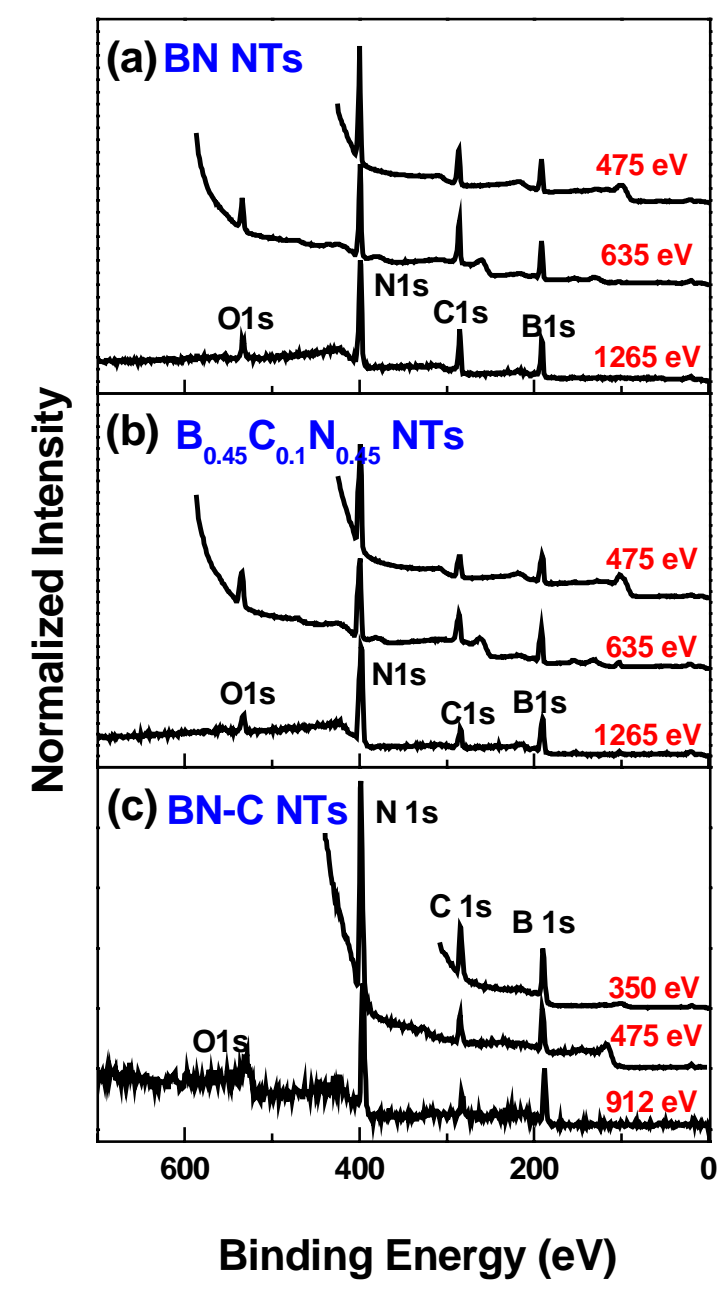

Figure S1: XPS survey scan spectrum measured using variable photon energies for the (a) BN NTs, (b) $\mathrm{B}_{0.45} \mathrm{C}_{0.1} \mathrm{~N}_{0.45} \mathrm{NTs}$, and (c) BN-C NTs. 


\section{(2) Fine-scanned B and N 1s XPS of BN NTs}

The B $1 s$ spectrum of the BN NTs is displayed in Figure S2a. The sample consisted of pure BN NTs and CNTs. Three photon energies, 360, 625, and $1265 \mathrm{eV}$, were used to compare the electronic structure depending on the probing depth. For all three photon energies, an asymmetric band is observed centered at $191 \mathrm{eV}$. From the curve fitting, this band can be deconvoluted into two bands at 191.0 (PB1) and 192.5 (PB2) eV, respectively. The binding energy of the $\mathrm{B}$ atoms bonded to the $\mathrm{N}$ atoms would be expected to appear at a lower energy than that of the dangling bonds or defects that are usually bonded to more electronegative $\mathrm{O}$ atoms. Therefore, the strongest PB1 can be assigned to the $\mathrm{B}$ atoms binding to the $\mathrm{N}$ atoms, and the weaker PB2 corresponds to the $\mathrm{B}$ atoms binding to the $\mathrm{O}$ atoms, which is consistent with the XPS of the BCN films. The average area \% values of PB1 are 55, 54, and $61 \%$ at 475, 635, and $1265 \mathrm{eV}$, respectively. As the photon energy increases, the fraction of the B-N structure (PB1) tends to increase, indicating the formation of more perfect $\mathrm{BN}$ layers in the interior of the nanotubes. The area \% values are listed in Table $\mathrm{S} 1$.

Figure S2b displays the N $1 s$ spectrum of the BN NTs, showing a band centered at $398 \mathrm{eV}$. The asymmetric shape suggests that there are at least two main components, so the band has been deconvoluted into two bands at 397.8 (PN0) and $399.1 \mathrm{eV}$ (PN1), respectively. The peaks at the lower binding energy (PNO) can be assigned to the $\mathrm{N}$ atoms bonded to the $\mathrm{B}$ atoms in the $h$-BN framework. The peak at the higher binding energy (PN1) is assigned to N-O and/or graphite-like N-C bonds. The graphite-like N-C structures could originate from the N-doped CNTs impurities. 
Table S1. Area \% values of the deconvoluted bands from the XPS B, C, and N $1 s$ peaks for the BN-NTs

\begin{tabular}{|c|c|c|c|c|c|c|c|c|c|}
\hline & \multicolumn{6}{|c|}{ Photon energy (eV) } \\
\hline & & & & \multicolumn{2}{|c|}{$475 \mathrm{eV}$} & \multicolumn{2}{|c|}{$635 \mathrm{eV}$} & \multicolumn{2}{|c|}{1265} \\
\hline \multicolumn{4}{|c|}{ B:N } & \multicolumn{2}{|c|}{$1: 1$} & \multicolumn{2}{|c|}{$1: 1$} & \multicolumn{2}{|c|}{$1: 1$} \\
\hline & & $\begin{array}{l}\text { Bonding } \\
\text { structures }\end{array}$ & $\begin{array}{c}\text { Position } \\
(\mathrm{eV})\end{array}$ & $\begin{array}{l}\text { Width } \\
(\mathrm{eV})\end{array}$ & Area \% & $\begin{array}{l}\text { Width } \\
(\mathrm{eV})\end{array}$ & Area \% & $\begin{array}{l}\text { Width } \\
(\mathrm{eV})\end{array}$ & Area \% \\
\hline \multirow[t]{2}{*}{ B } & PB1 & B-N & 191.0 & 1.1 & 55 & 1.1 & 54 & 1.7 & 61 \\
\hline & PB2 & $\mathrm{B}-\mathrm{O}$ & 192.5 & 2.5 & 45 & 2.6 & 46 & 3.0 & 39 \\
\hline \multirow[t]{2}{*}{$\mathrm{N}$} & PNO & N-B & 397.8 & 1.2 & 57 & 1.2 & 57 & 1.7 & 64 \\
\hline & PN1 & $\begin{array}{c}\mathrm{N}-\mathrm{O} \text { (and } \\
\mathrm{N}-\mathrm{C})\end{array}$ & 399.1 & 2.6 & 43 & 2.3 & 43 & 3.4 & 36 \\
\hline
\end{tabular}

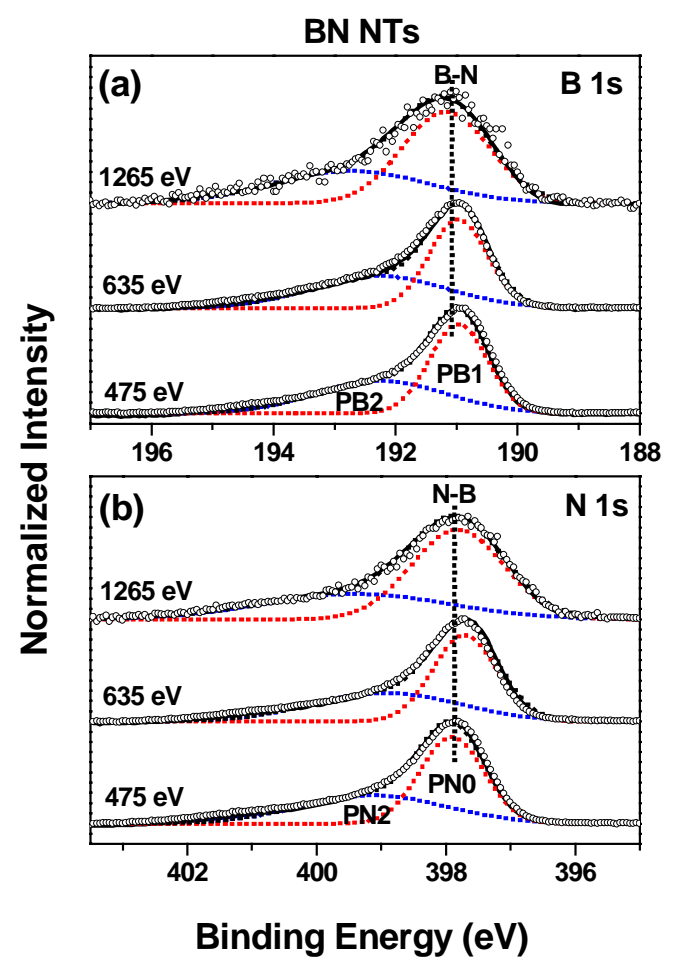

Figure S2: (a) The B $1 s$ XPS spectrum of the BN NTs using 360, 625, and $1265 \mathrm{eV}$. The data points (open circles) of the B $1 s$ band are fitted by two Voigt functions, PB1 and PB2 (dotted lines). (b) The N $1 s$ XPS spectrum of the BN NTs. The data points (open circles) are fitted by two Voigt functions, PN0 and PN1 (dotted lines). 


\section{(3) Survey- and fine-scanned XPS of $h$-BN powders}

Figure S3a displays the survey-scanned XPS of the $h$-BN powders (Aldrich, 99\%), showing the $\mathrm{C}$ contaminants. The photon energy is $625 \mathrm{eV}$. The $\mathrm{B} 1 \mathrm{~s}$ spectrum is displayed in Figure S3b. It shows an asymmetric band centered at $191 \mathrm{eV}$. From the curve fitting, the band can be deconvoluted into two bands at 189.7 (PB0) and 191.0 (PB1) eV, corresponding to the B-C and B-N bond structures, respectively. Figure S3c displays the C $1 s$ spectrum, showing an asymmetric band centered at $284 \mathrm{eV}$. This can be deconvoluted into two bands at 283.4 (PC0) and 284.6 (PC1) eV, which are assigned to the $\mathrm{C}-\mathrm{B}$ and $\mathrm{C}-\mathrm{C}$ bonding structures, respectively. Figure S3d corresponds to the $\mathrm{N} 1 \mathrm{~s}$ spectrum. The asymmetric band centered at $399 \mathrm{eV}$ has been deconvoluted into two bands at 397.5 (PN0) and 398.9 (PN1) eV, corresponding to the N-B and N-C structures, respectively. The area \% of each resolved band is listed in Table S2. The C contaminant can slightly influence the electronic states of the BN NTs, similarly to that of the BN-C NTs.

Table S2: Area \% values of the deconvoluted bands from the XPS B, C, and N $1 s$ peaks for the $h$-BN powders. The photon energy is $625 \mathrm{eV}$.

\begin{tabular}{|c|c|c|c|c|c|}
\hline \multirow{2}{*}{ Elements } & Band & $\begin{array}{c}\text { Bonding } \\
\text { Structures }\end{array}$ & $\begin{array}{c}\text { Position } \\
(\mathrm{eV})\end{array}$ & FWHM & Area (\%) \\
\hline \multirow{2}{*}{ B } & PB0 & B-C & 189.7 & 2.8 & 41 \\
\cline { 2 - 6 } & PB1 & B-N & 191.0 & 1.7 & 59 \\
\hline \multirow{2}{*}{$\mathbf{C}$} & PC0 & C-B & 283.4 & 3.2 & 49 \\
\cline { 2 - 6 } & PC1 & C-C & 284.6 & 1.9 & 51 \\
\hline \multirow{2}{*}{$\mathbf{N}$} & PN0 & N-B & 397.5 & 3.0 & 41 \\
\cline { 2 - 6 } & PN1 & N-C & 398.9 & 1.7 & 59 \\
\hline
\end{tabular}



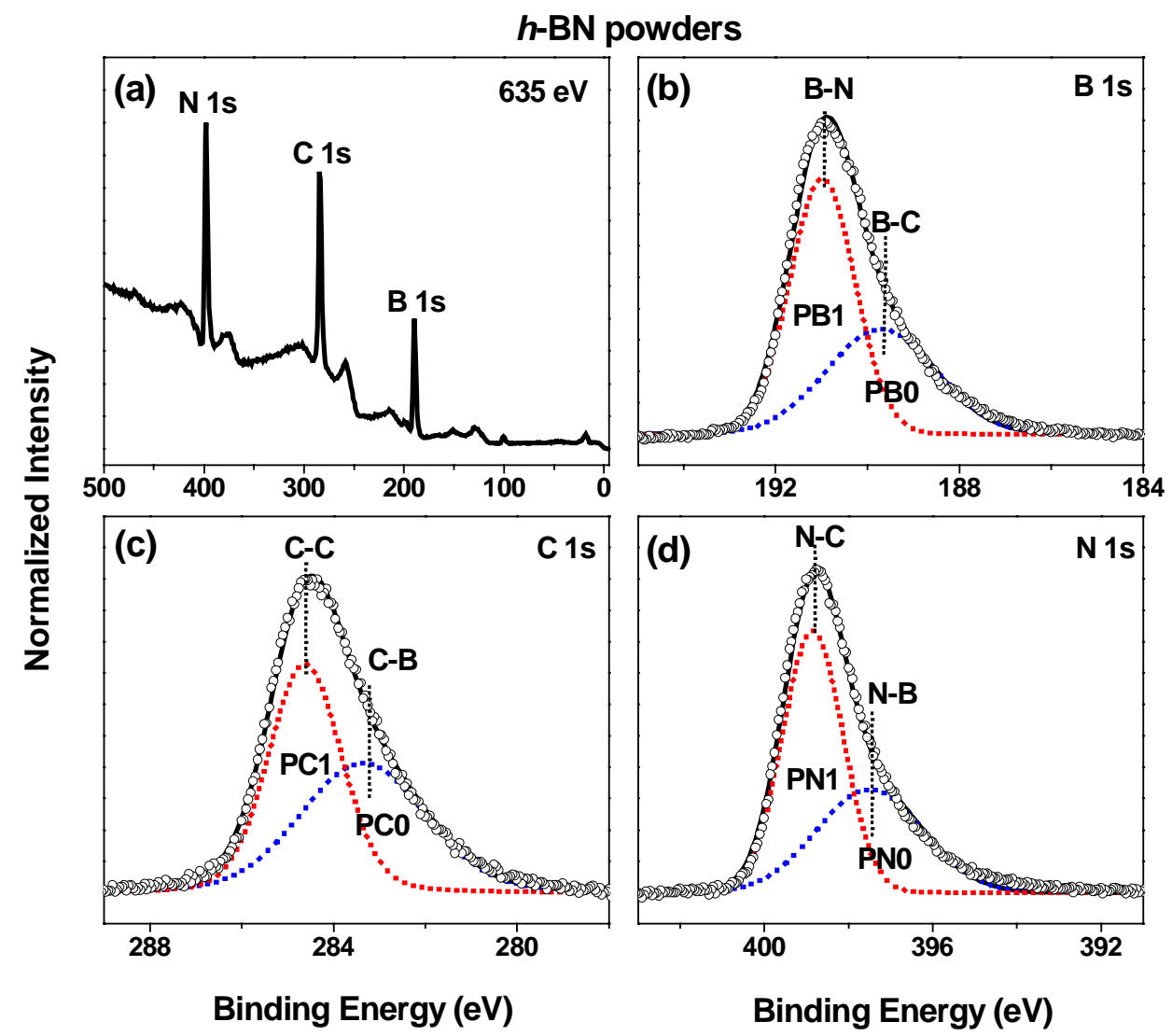

Figure S3: (a) XPS survey scan spectrum of the $h$-BN powders, measured using $625 \mathrm{eV}$, (b) Fine scanned B $1 s$ XPS spectrum. The data points (open circles) are fitted by two Voigt functions, PB0 and PB1 (dotted lines). (c) Fine-scanned C $1 s$ XPS spectrum. The data points (open circles) are fitted by two Voigt functions, PC0 and PC1 (dotted lines). (d) Fine-scanned N $1 s$ XPS spectrum. The data points (open circles) are fitted by two Voigt functions, PN0 and PN1 (dotted lines).

\section{(4) CL and PL of BN, $\mathrm{B}_{0.45} \mathrm{C}_{0.1} \mathrm{~N}_{0.45}$ and $\mathrm{BN}-\mathrm{C}$ NTs}

Figure S4a displays the room-temperature CL spectrum of the BN NTs, $\mathrm{B}_{0.45} \mathrm{C}_{0.1} \mathrm{~N}_{0.45}$ NTs, and BN-C NTs. The BN NTs show a broad emission band at 3.75 
eV. While the BN-C NTs exhibit the same PL feature as that of the BN NTs, the $\mathrm{B}_{0.45} \mathrm{C}_{0.1} \mathrm{~N}_{0.45}$ NTs exhibit a red shifted band at $3.5 \mathrm{eV}$. The efficient formation of the B$\mathrm{C}$ and $\mathrm{C}-\mathrm{N}$ bonds in the $\mathrm{B}_{0.45} \mathrm{C}_{0.1} \mathrm{~N}_{0.45}$ NTs would shift the peak position to the lower energy region. The efficient formation of the $\mathrm{B}-\mathrm{C}$ and $\mathrm{C}-\mathrm{N}$ bonds in the $\mathrm{B}_{0.45} \mathrm{C}_{0.1} \mathrm{~N}_{0.45}$ NTs would shift the peak position to the lower energy region. We suggest that this emission originated from the band edge (e.g. donor-acceptor-pair recombination), and the red shift supports the decrease in the band gap. The peak at $\sim 2 \mathrm{eV}$ would be originated from the defects and impurities.

There are a number of works on the CL emission of BN NTs and BCN NTs, showing the inconsistent position of the band edge emission. Zhi et al. reported the room-temperature CL emission at $3.1 \mathrm{eV}$ from the BN NTs, and explained it by there being the possibility of a low band gap or B- and N-vacancy-type defect-trapped states [Zhi, C.; Bando, Y.; Tang, C.; Golberg, D.; Xie, R.; Sekigushi, T. Appl. Phys. Lett. 2005, 86, 213110]. In contrast, the CL of the individual porous BCN NT (B:C:N 1:1:1) showed the band edge emission at $3.81 \mathrm{eV}$ (room temperature) and $3.89 \mathrm{eV}(20 \mathrm{~K})$ [Ref. 15h: Yin, L. -W.; Bando, Y.; Golberg, D.; Gloter, A.; Li, M. -S.; Yuan, X.; Sekiguchi, T. J. Am. Chem. Soc. 2005, 127, 16354]. The CL emission of the present $\mathrm{B}_{0.45} \mathrm{C}_{0.1} \mathrm{~N}_{0.45} \mathrm{NTs}$ appears in a lower energy region than that of these BCN NTs.

The resolved peak from the PL of the $\mathrm{BN}$ and $\mathrm{B}_{0.45} \mathrm{C}_{0.1} \mathrm{~N}_{0.45} \mathrm{NTs}$ at $7 \mathrm{~K}$, excited by $3.815 \mathrm{eV}$ (Figure S4b). The PL spectrum of the BN-C NTs shows exactly the same feature as that of the BN NTs, and so is not displayed here. The PL spectra exhibit a few $h$-BN LO phonon replicas at $3.29,3.13$, and $2.95 \mathrm{eV}$, separated by $\sim 0.17 \mathrm{eV}$, corres ponding to LO phonon $\left(1370 \mathrm{~cm}^{-1}\right)$ replicas (marked by the dotted line). 
Consistently with the CL spectrum, the $\mathrm{B}_{0.45} \mathrm{C}_{0.1} \mathrm{~N}_{0.45}$ NTs exhibit a broader PL feature in the lower energy region, compared to that of the BN NTs, which can be correlated with the decrease in their band gap. The phonon replicas of the room-temperature PL $\mathrm{s}$ pectrum were reported by the Zettl group [Wu, J.; Han, W. -Q.; Walukiewicz, W.; Ager III, J. -W.; Shan, W.; Haller, E. E.; Zettl, A. Nano Lett. 2004, 4, 647]. The PL, excited by $5.08 \mathrm{eV}$, consists of an emission band with a number of phonon replicas at $\sim 4,3.83$, and $3.66 \mathrm{eV}$ for both the $\mathrm{BN}$ and BCN NTs.

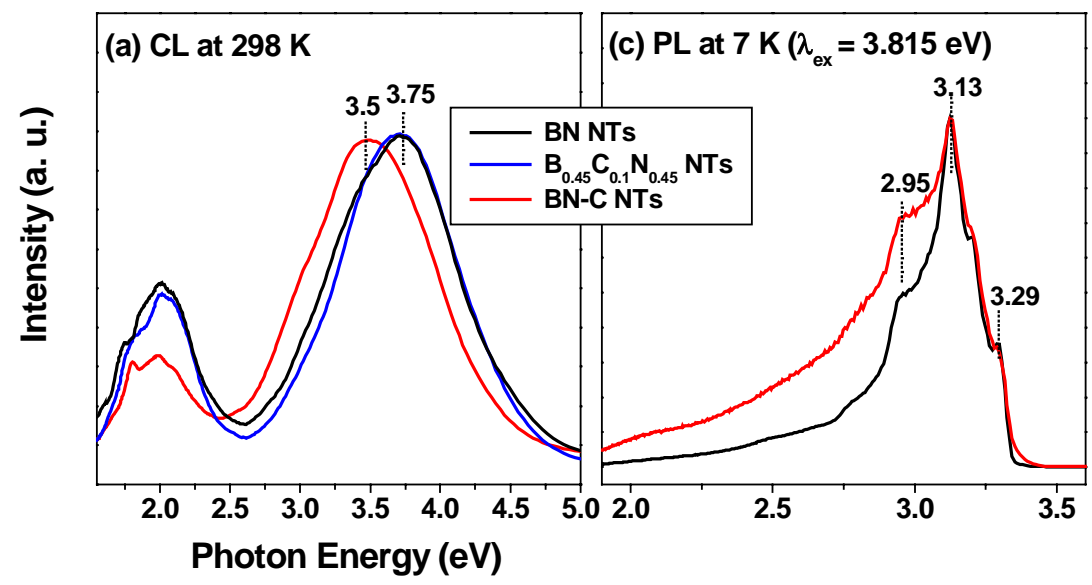

Figure S4: (a) Room-temperature (298 K) CL spectrum (excited by $10 \mathrm{kV}$ ) of BN NTs, $\mathrm{B}_{0.45} \mathrm{C}_{0.1} \mathrm{~N}_{0.45}$ NTs, and BN-C NTs. (b) PL spectra measured at $7 \mathrm{~K}$, excited by $325 \mathrm{~nm}$ (3.815 eV) He-Cd laser. The series of LO phonon replicas separated by $\sim 0.17 \mathrm{eV}$ are marked by dotted lines. 


\section{(5) Raman spectrum of $\mathrm{BN}, \mathrm{B}_{0.45} \mathrm{C}_{0.1} \mathrm{~N}_{0.45}$, and $\mathrm{BN}-\mathrm{C}$ NTs at room temperature}

Figure S5 displays the Raman spectra of the BN NTs, $\mathrm{B}_{0.45} \mathrm{C}_{0.1} \mathrm{~N}_{0.45} \mathrm{NTs}$, and BN-C NTs measured at room temperature. The BN NTs show a strong band at $1363 \mathrm{~cm}^{-1}$, which is assigned to the Raman active mode, $\mathrm{E}_{2 \mathrm{~g}}$, derived from the in-plane atomic displacement of the $\mathrm{B}$ and $\mathrm{N}$ atoms toward each other [Nemanich, R. J.; Solin, S. A.; Martin, R. M. Phys. Rev. B 1981, 23, 6348]. The full-width at half maximum (FWHM) is $38 \mathrm{~cm}^{-1}$. For the $\mathrm{B}_{0.45} \mathrm{C}_{0.1} \mathrm{~N}_{0.45} \mathrm{NTs}$, the peak position and the FWHM remain nearly the same as those of the BN NTs. However, another band arises at $1585 \mathrm{~cm}^{-1}$, which originates from the Raman active in-plane atomic displacement $E_{2 g}$ mode of the graphite sheets $(G$ band). The broadening of the base part of the $1363 \mathrm{~cm}^{-1}$ band (BN band) could be due to the contribution of the disordered graphite sheets ( $\mathrm{D}$ band) that usually appears broadly at $\sim 1350 \mathrm{~cm}^{-1}$. The $\mathrm{C}$ outerlayers of the BN-C NTs increase significantly the FWHM of the BN peak and also the intensity of the G band. The increase in the FWHM of the BN band is likely caused by the D band. The unchanged position of the BN peak at 1363 $\mathrm{cm}^{-1}$ may be related to the $\mathrm{C}$ domains segregated from the $\mathrm{BN}$ domains. These results show that Raman spectroscopy can be one of the most powerful tools to distinguish the homogeneously C-doped BCN NTs from the C-outerlayered BN NTs. 


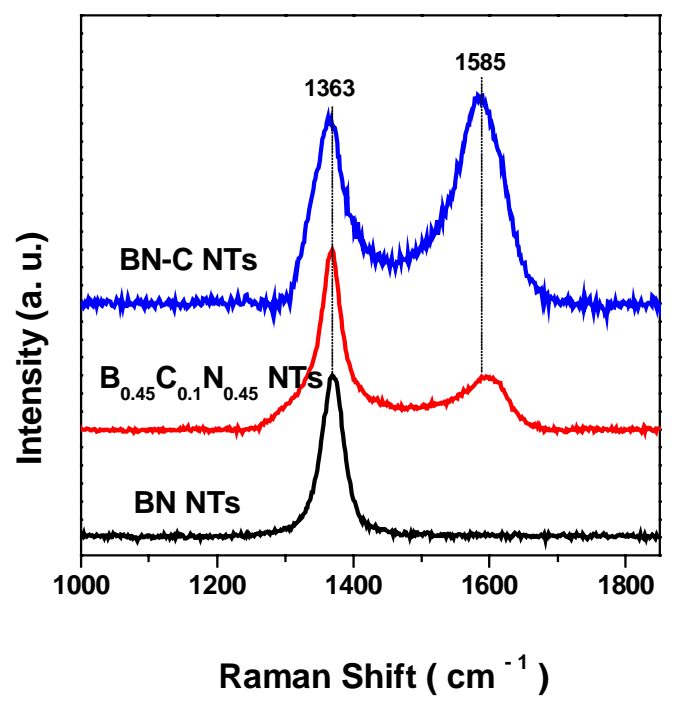

Figure S5: Raman spectra of the BN NTs, $\mathrm{B}_{0.45} \mathrm{C}_{0.1} \mathrm{~N}_{0.45} \mathrm{NTs}$, and BN-C NTs. The excitation source is a $514.5 \mathrm{~nm}$ argon ion laser.

\section{(6) Structure of " $q$-motif"}

The $q$-motif consists of three contiguous carbon rings substitutionally embedded in a BN NT, with central pyridine ring originally bonded to $B_{d}$. Two other rings originally bonded to $\mathrm{B}_{\mathrm{d}}$ are not substituted by $\mathrm{C}$ atoms, thus not forming pyridine rings. These two rings are stabilized by the formation of an $\mathrm{N}-\mathrm{N}$ bond between them. It is reasonable to believe that it represents one of the best pyridine-like motives in BCN NTs other than the $p$-motif, since it has contiguous carbon rings. One $q$-motif has exactly the same numbers of $\mathrm{B}$ and $\mathrm{N}$ atoms substituted by $\mathrm{C}$ atoms as a $p$-motif. A detailed calculation of the energies of the $p$ - and $q$-motives in a supercell of the same size (= four primitive cells of the BN NT) indicates that the former is substantially more stable than the latter, 
as shown by the energy difference of $1.23 \mathrm{eV}$. In short, the $p$-motif will presumably represent the best pyridine-like local structure of the BCN NTs.

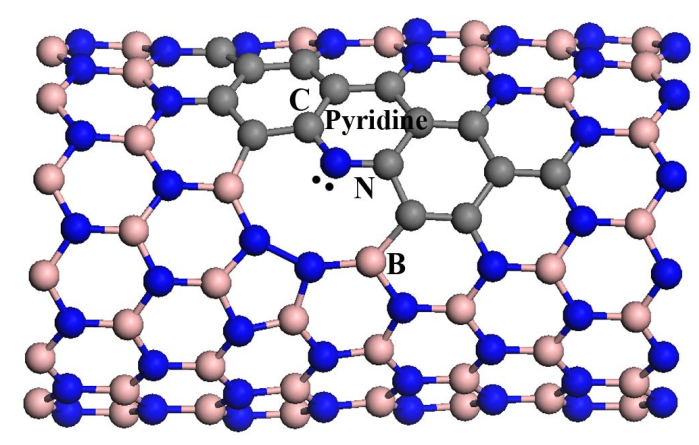

Figure S6: A local $q$-motif in a supercell of four primitive cells of $(12,0)$ BN NT. Carbon atoms are in black color. 